\title{
Protocol-driven prevention of perioperative hypothermia in the pediatric neurosurgical population
}

\author{
Ian Mutchnick, MD, MS, $, 1,2$ Meena Thatikunta, MD, ${ }^{2}$ Julianne Braun, MSN, APRN, ${ }^{1}$ \\ Martha Bohn, BSN, RN, CNOR, ${ }^{3}$ Barbara Polivka, PhD, RN, ${ }^{4}$ Michael W. Daniels, MS, ${ }^{5}$ \\ Rachel Vickers-Smith, PhD, MPH, ${ }^{6}$ William Gump, MD, ${ }^{1,2}$ and Thomas Moriarty, MD, PhD ${ }^{1,2}$

\begin{abstract}
${ }^{1}$ Division of Pediatric Neurosurgery, Norton Children's Hospital/Norton Neuroscience Institute, Louisville; '2Department of Neurosurgery, University of Louisville; ${ }^{3}$ Division of Operative Services, Norton Children's Hospital, Louisville, Kentucky; ${ }^{4}$ University of Kansas School of Nursing, Kansas City, Kansas; ${ }^{5}$ Department of Bioinformatics and Biostatistics, School of Public Health and Information Sciences, University of Louisville; and ' $U$ University of Louisville School of Nursing, Louisville, Kentucky
\end{abstract}

OBJECTIVE Perioperative hypothermia $(\mathrm{PH})$ is a preventable, pathological, and iatrogenic state that has been shown to result in increased surgical blood loss, increased surgical site infections, increased hospital length of stay, and patient discomfort. Maintenance of normothermia is recommended by multiple surgical quality organizations; however, no group yet provides an ergonomic, evidence-based protocol to reduce $\mathrm{PH}$ for pediatric neurosurgery patients. The authors' aim was to evaluate the efficacy of a $\mathrm{PH}$ prevention protocol in the pediatric neurosurgery population.

METHODS A prospective, nonrandomized study of 120 pediatric neurosurgery patients was performed. Thirty-eight patients received targeted warming interventions throughout their perioperative phases of care (warming group-WG). The remaining 82 patients received no extra warming care during their perioperative period (control group-CG). Patients were well matched for age, sex, and preparation time intraoperatively. Hypothermia was defined as $<36^{\circ} \mathrm{C}$. The primary outcome of the study was maintenance of normothermia preoperatively, intraoperatively, and postoperatively.

RESULTS WG patients were significantly warmer on arrival to the operating room (OR) and were $60 \%$ less likely to develop PH ( $p$ < 0.001). Preoperative forced air warmer use both reduced the risk of $\mathrm{PH}$ at time 0 intraoperatively and significantly reduced the risk of any PH intraoperatively $(p<0.001)$. All patients, regardless of group, experienced a drop in core temperature until a nadir occurred at 30 minutes intraoperatively for the WG and 45 minutes for the CG. At every time interval, from preoperatively to 120 minutes intraoperatively, CG patients were between 2 and 3 times more likely to experience $\mathrm{PH}(\mathrm{p}<0.001)$. All patients were warm on arrival to the postanesthesia care unit regardless of patient group.

CONCLUSIONS Preoperative forced air warmer use significantly increases the average intraoperative time 0 temperature, helping to prevent a fall into $\mathrm{PH}$ at the intraoperative nadir. Intraoperatively, a strictly and consistently applied warming protocol made intraoperative hypothermia significantly less likely as well as less severe when it did occur. Implementation of a warming protocol necessitated only limited resources and an OR culture change, and was well tolerated by OR staff.

https://thejns.org/doi/abs/10.3171/2019.12.PEDS1980

KEYWORDS perioperative hypothermia; normothermia; pediatric neurosurgery; pediatric surgery; warming

$\mathrm{P}$ ERIOPERATIVE hypothermia (PH) was first described in 1847 by German physicians Ernst von Bibra and Emil Harlass, noting that core temperatures in rabbits receiving sulfuric ether dropped to deeply subphysiological levels (see Bräuer). ${ }^{5}$ Given the considerable challenges facing anesthesia at the time, PH did not receive much direct attention and was considered "a normal consequence of surgery;" a concept that remained intact into the 1980s when Daniel Sessler began his anesthesia training and began to look into the effects of $\mathrm{PH}$ on surgical patients. ${ }^{18,25}$ In a landmark paper in the New England Journal of Medicine in 1996, Kurz et al. found surgical site infections (SSIs) in 18 of $96(19 \%)$ patients receiving routine intraoperative thermal care, whereas only 6 of 104

ABBREVIATIONS CG = control group; CMS = Centers for Medicare and Medicaid Services; FAW = forced air warmer; NPr = normothermia protocol; NSQIP = National Surgical Quality Improvement Project; OR = operating room; PACU = postanesthesia care unit; $\mathrm{PH}=$ perioperative hypothermia; $\mathrm{RWL}=$ radiant warming light; SCIP = Surgical Care Improvement Project; SSI = surgical site infection; WG = warming group.

SUBMITTED February 10, 2019. ACCEPTED December 2, 2019.

INCLUDE WHEN CITING Published online February 14, 2020; DOI: 10.3171/2019.12.PEDS1980. 
(6\%) patients receiving additional warming had SSIs $(\mathrm{p}=$ 0.009). ${ }^{15}$ Additionally, patients receiving routine thermal care had their length of stay prolonged 2.6 days (approximately 20\%) versus those kept warm intraoperatively ( $p$ $=0.01$ ). Rajagopalan et al. reviewed the available literature on estimated blood loss and need for transfusion as a function of $\mathrm{PH}$; they found that $\mathrm{PH}$ of even $<1^{\circ} \mathrm{C}$ was significantly correlated with a $16 \%$ increase in estimated blood loss and raised the risk for transfusion by approximately $22 \%$. They concluded that "maintaining perioperative normothermia reduces blood loss and transfusion requirement by clinically important amounts." ${ }^{20}$ Arising from these consistently reported relationships, the maintenance of perioperative euthermia has been incorporated into multiple guidelines promoting safe surgery, including those produced by the Centers for Medicare and Medicaid Services (CMS) Surgical Care Improvement Project (SCIP), the National Surgical Quality Improvement Project (NSQIP), the WHO Surgical Safety Checklist, and the National Quality Forum. 2,24,27

Despite both data and guidelines, there are currently no validated protocols for ensuring perioperative normothermia in the pediatric neurosurgical population-and pediatric surgical patients appear to be at significant risk of $\mathrm{PH}$. Two large studies reported relatively high rates of PH in the pediatric surgical population: Beedle et al. documented a PH rate of approximately $20 \%$ in 1504 patients, and Pearce et al. reported a rate of $\mathrm{PH}$ of $53 \%$ on 530 pediatric patients. ${ }^{119}$ Although Kim et al. found that only $6 \%$ of pediatric neurosurgery patients were hypothermic on arrival to the postanesthesia care unit (PACU), this is misleading because patients are least likely to be hypothermic at the end of a case given that standard operating room (OR) care involves a forced air warmer (FAW) and operative draping provides a good deal of insulation. ${ }^{14}$ Not only are patients at significant risk, but current OR culture may not even prioritize a workflow fostering perioperative normothermia: a Europe-wide survey indicated that a mere $40 \%$ of patients were warmed intraoperatively and temperature was measured in only $20 \%$ of cases. ${ }^{28}$ Although perhaps underutilized, interventions to prevent $\mathrm{PH}$ appear to work well: with a normothermia protocol (NPr), Beedle et al. reduced their $\mathrm{PH}$ rate to $1.84 \%$, a finding echoed by multiple studies in multiple specialties. ${ }^{11,29,30}$ There are no studies to date focusing only on pediatric neurosurgery patients.

Given the effectiveness of $\mathrm{PH}$ prevention protocols, reasons for high rates of $\mathrm{PH}$ have been sought. Studies have documented deficits in nursing staffs' awareness regarding measurement and prevention of $\mathrm{PH}^{7}$ There are no studies regarding physician awareness of either the detriments of PH or its effective prevention. Both staff education and protocol implementation are clearly effective in changing OR staff behavior regarding PH. Workflow changes can also be effective: requiring documentation of temperature as well as the charting of $\mathrm{PH}$ interventions by OR staff and anesthesia providers increases measurement compliance. ${ }^{8,14}$ Factors limiting the implementation of PH prevention protocols may include availability and cost of equipment. ${ }^{8,27}$ Additionally, surgeon preference for cooler ambient temperature is a pervasive factor in the OR..$^{10,16}$
A compromise to keep the room warm during anesthesia induction and draping of the patient and then reduce the room temperature to a comfortable level thereafter is reasonable. ${ }^{16}$ At our institution, standard $\mathrm{PH}$ prevention for the pediatric neurosurgery service was limited to intermittent use of a radiant warming light (RWL) and the standard placement of an FAW on all patients prior to draping. As part of a quality improvement initiative, we wanted to find a set of actions that could effectively prevent $\mathrm{PH}$ without imposing an unacceptable ergonomic burden on the OR staff and physicians.

\section{Methods}

Institutional review board approval was obtained. We designed a nonrandomized, controlled, prospective study of 120 pediatric patients at Norton Children's Hospital. All patients undergoing a neurosurgical procedure between March 1, 2013, and August 1, 2013, were included. In order to reach statistical significance for the patients receiving the NPr, additional patients were added between October 1, 2013, and March 1, 2015. These patients were added more slowly and recruitment was dependent on having a specific OR staff team covering surgeon 1's patients-a team that was willing to implement the NPr given staff turnover and need for training. All patients operated on by surgeon 1 received this new $\mathrm{NPr}$ (detailed below) and are referred to as the warming group (WG). All patients operated on by surgeons 2 and 3 received the institutional standard protocol: many patients received intermittent RWL during preparation; all received an FAW prior to draping. These patients are referred to as the control group (CG). Reaching consensus among the surgeons for the study was not difficult; they were satisfied with their respective roles for the study and thought that practice might be improved with clear information. Consensus among the OR staff for the study was not difficult either; quality improvement projects were not new and there was motivation to find ways to reduce $\mathrm{PH}$ given its prominence among the newer sets of surgery quality guidelines.

The NPr was developed by a team including the lead investigator, an OR circulating nurse, and the nurse manager for the neurosurgical service. They used the best available evidence for maintaining perioperative euthermia in the pediatric population with an eye toward workplace ergonomic viability. In the preoperative period, patients' initial temperature was recorded with a tympanic thermometer (Welch Allyn Braun Thermoscan Pro 6000) and then every 15 minutes thereafter. Tympanic thermometry has been shown to be as accurate as mercury-based axillary temperatures, but has a $95 \% \mathrm{CI}$ of $2.62^{\circ} \mathrm{C}$ around core temperature measurements. ${ }^{6,21}$ Despite its shortcomings, we thought that this method was an adequate balance between invasiveness and precision in the pre- and postoperative patient phases of care.

Temperature was measured immediately prior to OR transfer. Families were educated on the importance of warming in order to enhance their participation. Patients had an FAW (Bair Hugger system; 3M) placed preoperatively. Intraoperatively, the OR temperature was set to $75^{\circ} \mathrm{F}$ at least 15 minutes prior to patient arrival, and an 
TABLE 1. Demographics and procedure types in 120 patients in the PH study

\begin{tabular}{lccc}
\hline \multicolumn{1}{c}{ Variable } & WG, $\mathrm{n}=38$ & $\mathrm{CG}, \mathrm{n}=82$ & $\mathrm{p}$ Value \\
\hline Mean age, yrs (SD) & $8.16(5.21)$ & $8.51(5.35)$ & 0.732 \\
\hline Sex, no. $(\%)$ & & & \\
\hline Male & $22(57.9 \%)$ & $39(47.6 \%)$ & $\mathrm{NS}$ \\
\hline Female & $16(42.1 \%)$ & $43(52.4 \%)$ & $\mathrm{NS}$ \\
\hline Mean time in OR, mins (SD) & $218(128)$ & $172(89)$ & $\mathbf{0 . 0 2 4}$ \\
\hline Procedure type, no. (\%) & & & \\
\hline Chiari & $6(15.8 \%)$ & $13(15.9 \%)$ & $\mathrm{NS}$ \\
\hline Craniotomy & $2(5.3 \%)$ & $1(1.2 \%)$ & $\mathrm{NS}$ \\
\hline Cranioplasty & $2(5.3 \%)$ & $4(4.9 \%)$ & $\mathrm{NS}$ \\
\hline Tethered cord release & $15(39.5 \%)$ & $51(62.2 \%)$ & $\mathbf{0 . 0 3 3}$ \\
\hline VNS & $4(10.5 \%)$ & $8(9.8 \%)$ & $\mathrm{NS}$ \\
\hline Other & $9(23.7 \%)$ & $5(6.1 \%)$ & $\mathrm{NS}$ \\
\hline
\end{tabular}

NS = not significant; VNS = vagal nerve stimulator.

Boldface type indicates statistical significance.

RWL was placed over the bed prior to patient arrival and was carefully maintained over the patient during anesthesia induction, surgical preparation, and draping. An FAW was placed and carefully covered with blankets to avoid altering circulating air patterns in a way that might increase the risk of infection. ${ }^{17}$ Temperature was measured intraoperatively via esophageal probe every 15 minutes and recorded by anesthesia staff. Postoperatively, 3 blankets were placed on the patient, making sure to cover the patient's head; the room temperature was returned to $75^{\circ} \mathrm{F}$; and an RWL was placed over the patient during undraping and transfer to the hospital bed. Temperature was measured via tympanic thermometer on arrival to the PACU. Temperature was recorded by nursing and anesthesia staff at the required time points per protocol. Nursing staff recorded adherence to each step of the NPr as it was done, and this form was collected for each patient in the WG.

\section{Statistical Analysis}

The intervention and control groups were compared on descriptive, procedure-related characteristics and hypothermic burden (area under the curve) by using independent-sample t-tests for continuous variables and chisquare and Fisher's exact tests for categorical variables.

\section{Results}

The WG included 38 patients and the CG included 82 patients. The average age at surgery was 8 years for both groups and was not statistically different; there were no significant sex differences between groups. There was a statistically significant difference in the average total operating time for the WG versus the CG: 218 minutes versus 172 minutes, respectively $(\mathrm{p}=0.024)$. Type of procedure was also analyzed between groups: the CG had a higher percentage of simple tethered cord releases $(62 \%$ vs $40 \%, \mathrm{p}=0.03$ ), but otherwise there were no significant differences between the 2 groups (Table 1). The difference

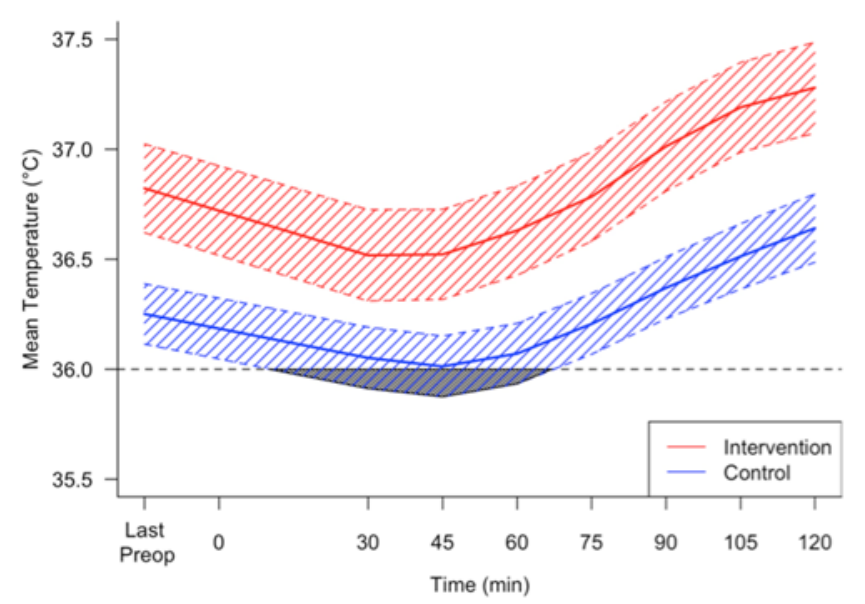

FIG. 1. Graph showing the mean temperatures at each time point from the last preoperative minute to 120 minutes intraoperatively with $95 \% \mathrm{Cl}$ band. WG patients in red, CG patients in blue. WG patients were significantly warmer on arrival to the OR $(p<0.001)$. Both groups had similar drops in temperature before normalizing, with temperature minimums occurring nearly 45 minutes into surgery. The lower $95 \% \mathrm{Cl}$ band never drops into the hypothermic region (dashed line at $36^{\circ} \mathrm{C}$ ) for the WG, unlike the CG. Figure is available in color online only.

in the number of "other" cases was across a small number of total cases and represents a variety of procedure types, making the $24 \%$ versus $6 \%$ difference statistically insignificant. Nursing compliance with the various parts of the NPr, prospectively recorded as part of total data collection, was $>95 \%$.

Figure 1 shows the mean temperatures at each time point, from the last preoperative temperature to $120 \mathrm{~min}$ utes intraoperatively, with a $95 \%$ CI. Both the CG and the WG had a similar drop in temperature, with minimums occurring near 45 minutes intraoperatively. The average time between entry into the OR and incision was 56 minutes for the CG and 57 minutes for the WG. The WG had a higher mean temperature at intraoperative time 0 as compared to the CG: $36.8^{\circ} \mathrm{C}$ versus $36.25^{\circ} \mathrm{C}$ ( $\mathrm{p}<0.001$ ). The lowest mean temperature for the groups was slightly different: $36.5^{\circ} \mathrm{C}$ at 30 minutes for the WG and $36.0^{\circ} \mathrm{C}$ at 45 minutes for the $\mathrm{CG}$.

Figure 2 was generated using a generalized linear mixed model with a random intercept for subjects evaluated for group effects over time. A model fit of controlling variables (age, preparation time) and linear and quadratic time effects was determined by the Akaike information criterion. By controlling these variables for patient age and preparation time, it emphasizes the effect of the intervention regardless of the variance in those controlled variables, as well as minimizing the statistical effects of individual starting temperatures. This model provides an estimated proportion of patients who became hypothermic at each time point, from the last preoperative temperature to 120 minutes intraoperatively. Any missing data were imputed using average group values for temperature and preparation time and then carried forward to future times. At every time interval CG patients were between 2 and 3 times more likely to experience $\mathrm{PH}$. 


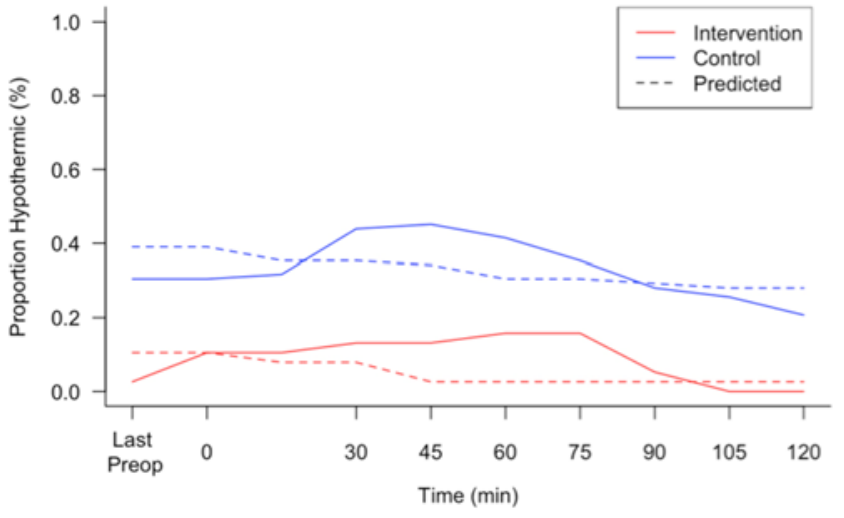

FIG. 2. Proportion of patients below $36.0^{\circ} \mathrm{C}$ at each time period from the last preoperative minute to 120 minutes intraoperatively. WG patients in red, CG patients in blue. The estimated proportion of patients experiencing $\mathrm{PH}$ at each time point was generated using a generalized linear mixed model with a random intercept for subjects evaluated for the group effects over time. A model fit of controlling variables (age, preparation time) and linear and quadratic time effects was determined by the Akaike information criterion. By controlling these variables for patient age and preparation time, the effect of the NPr is emphasized, regardless of the variance in age, preparation time, or individual starting temperatures. Any missing data were imputed using average group values for temperature and preparation time and then carried forward to future times. At every time interval CG patients were between 2 and 3 times more likely to experience $\mathrm{PH}$ as compared to the WG. Figure is available in color online only.

Figure 3 shows a statistically rigorous calculated aggregate of both the likelihood of experiencing $\mathrm{PH}$ and the degree of $\mathrm{PH}$ experienced by the WG and $\mathrm{CG}$ at each time point - a combination of both the likelihood of being cold and how cold each group was at each time point. The perioperative hypothermic burden is calculated by taking the total amount of hypothermia in each group at each time point (the sum of all temperature differences $<36^{\circ} \mathrm{C}$ at each time point) and normalizing this to the proportion of patients who were hypothermic at that time point by dividing that total hypothermic deficit by the number of patients at risk for $\mathrm{PH}$ in that group at that time point. The WG had a significantly $(p=0.00002)$ lower perioperative hypothermic burden than the CG throughout the perioperative time frame. All patients were warm on arrival to the PACU regardless of patient group. Statistical analysis of this time period was not carried out.

Although no formal data on staff contentment with the NPr was collected, surgeon 1 was present for all cases in which this protocol was used. Although the staff mentioned at times how warm the room was, at no time did any of them require a heat-related break. Additionally, once the justification for the warming measures was explained, staff were strongly in support of the NPr, with a solid sentiment that better patient care was being delivered.

\section{Discussion}

In a group of 120 pediatric patients, 38 patients received targeted warming interventions throughout their perioperative (pre-, intra-, and postoperative) phases of care. The

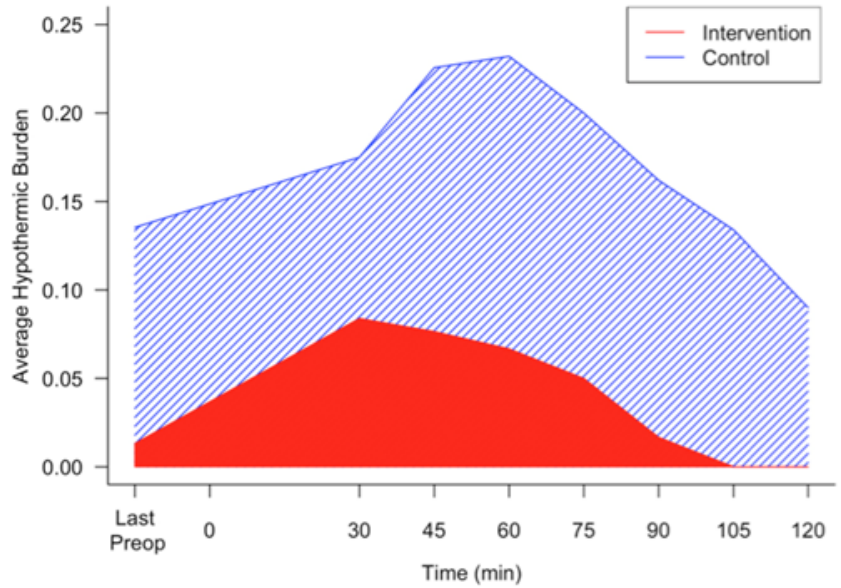

FIG. 3. Perioperative hypothermic burden at each time period from the last preoperative minute to 120 minutes intraoperatively. WG patients in red, CG patients in blue. The so-called hypothermic burden is a statistically rigorous calculated aggregate of both the likelihood of experiencing $\mathrm{PH}$ and the degree of $\mathrm{PH}$ experienced by the WG and $\mathrm{CG}$ at each time point-a combination of both the likelihood of being cold and how cold each group was at each time point. The perioperative hypothermic burden is calculated by taking the hypothermic deficit at each time point (the sum of all temperature differences $<36^{\circ} \mathrm{C}$ at each time point) and dividing the total hypothermic deficit by the number of patients at risk for PH in that group at that time point. The WG had a significantly $(p=$ 0.00002 ) lower perioperative hypothermic burden than the CG throughout the perioperative time frame. Figure is available in color online only.

remaining 82 patients received no extra warming care during their perioperative period, beyond the standard measures described above. The WG and CG were well matched for age at surgery and sex. There was a significant difference in average procedure time -218 minutes for the WG versus 172 minutes for the CG ( $p=0.024)$. Although our data did not provide an explanation for this difference, we believed that this was probably the result of surgeon 1 being the most junior surgeon of the three; data collection took place during his 2nd postfellowship year, whereas the senior surgeon was in his 2 nd decade of practice. The only other significant difference between experimental groups was the type of procedure: the CG had a higher percentage of simple tethered cord releases than did the WG (62\% vs $40 \%, \mathrm{p}=0.03$ ); no reason could be found for this. Other than for tethered cords, the WG and CG were well matched for procedure type.

There was a significant $(\mathrm{p}<0.001)$ difference in the mean temperatures between WG and $\mathrm{CG}$ at all time points. The thermodynamic trends for each group were, however, similar: the mean temperature for both CG and WG patients fell from intraoperative time 0 until their nadir, falling toward the end of the preparation period. The precise time to lowest mean temperature for the groups was slightly different: $36.5^{\circ} \mathrm{C}$ at 30 minutes for the WG and $36.0^{\circ} \mathrm{C}$ at 45 minutes for the CG. Despite there being a significant difference in the number of tethered cords, the average preparation time (from OR entry to incision) was 56 minutes for the CG and 57 minutes for the WG. Preparation time presents a particular risk of $\mathrm{PH}$ to our 
patients. Anesthesia dampens thermal homeostatic responses in patients. Normally, a drop of $0.2^{\circ} \mathrm{C}$ will trigger a thermoregulatory response; with anesthesia the average patient must experience a $0.6^{\circ} \mathrm{C}-0.8^{\circ} \mathrm{C}$ drop in core temperature to trigger this response. Anesthesia also blunts peripheral vasoconstriction effectively transferring heat from core to periphery, and reduces metabolic heat production by $15 \%-30 \%$, rendering patients less able to maintain adequate core temperature. ${ }^{3,12,23}$ Pediatric patients are at particular risk for $\mathrm{PH}$ due to the following: a high surface area to body mass ratio, increased heat loss from the head, smaller stores of fat, and a less effective thermoregulatory mechanism. ${ }^{14}$ Additionally, the work of breathing contributes to a significant amount of heat production in neonates and is nearly eliminated during general anesthesia. ${ }^{10}$ Nonshivering thermogenesis, an important method of heat production for neonates, is impaired by anesthesia. ${ }^{10,23}$

Protecting patients during the preparatory phase of intraoperative care would appear to be the key to maintaining euthermia perioperatively. Once the patient is draped and surgery is under way, they were well insulated and covered with an FAW regardless of which group they were in. Although CG patients had a larger PH deficit to recover from, all patients begin to warm up by intraoperative time 45 minutes. The first tool for protecting patients from $\mathrm{PH}$ is preoperative FAW, and 2 issues appear to be important. First, preoperative FAW use reduces the risk of $\mathrm{PH}$ at time 0 intraoperatively. Note Fig. 2, in which the probability of being hypothermic at time 0 intraoperatively was only approximately 0.1 for WG patients, versus approximately 0.3 for the CG. Additionally, preoperative FAW appears to facilitate patient arrival to the OR with a higher core temperature, helping to insulate patients from $\mathrm{PH}$ during the initial intraoperative decline in core temperature that all patients face, regardless of whether they receive a warming intervention. Figure 2 suggests that careful attention to patient euthermia during the preparation phase intraoperatively is also important. Patients in the CG were significantly more likely to experience $\mathrm{PH}$; within $15 \mathrm{~min}$ utes of arrival to the OR, patients in the CG experienced a $33 \%$ increase in their risk of experiencing $\mathrm{PH}$. At no point intraoperatively did WG patients experience a risk of $\mathrm{PH}>$ 0.2 ; CG patients never saw a risk of $\mathrm{PH}<0.3$.

The incidence of pediatric neurosurgical PH is not well known, but our results suggest that without a carefully maintained NPr, $50 \%$ of pediatric neurosurgery patients will experience $\mathrm{PH}$, defined as a core temperature $<36^{\circ} \mathrm{C}$. This incidence is similar to the results obtained by Pearce et al., who quoted a $52 \%$ rate of pediatric intraoperative hypothermia, defined by their group as an intraoperatively measured temperature $<36^{\circ} \mathrm{C}$ for more than 5 minutes. ${ }^{19}$ Using our NPr measures, we were able to reduce that incidence to approximately $20 \%$ in the WG as well as dramatically reduce the degree to which a given patient with $\mathrm{PH}$ was hypothermic - not only were patients less likely to be cold, but also they were less cold. Intuitively presented in Fig. 3, significant differences in the average hypothermic burden start almost immediately after OR arrival, and peak at approximately 1 hour into intraoperative time.

Even at $20 \%$, our incidence of hypothermia in the WG is not optimal-that rate would be $0 \%$. This demands refinement of the warming protocols-either to alter the protocol or to improve adherence. To improve on the first iteration of our NPr, we have made further changes since generating the data reported in this paper. We have created a culture in which patient temperatures during the preparatory phase of OR care are more explicitly monitored; anesthesia staff have become used to alerting the surgeons to drops in temperature. If a patient's temperature is falling, we preferentially opt to increase the room temperature because a high-enough ambient temperature will obviate $\mathrm{PH} .{ }^{22}$ Finally, we have prioritized placing the FAW as early in the process of patient preparation as possible; in order to avoid possible increases in SSI due to air currents we completely cover the FAW before turning it on..$^{13}$ It is worth noting that of the 8 patients in the WG who had any episode of intraoperative hypothermia, 7 episodes occurred relatively early in our study and may reflect worse protocol adherence or implementation. It is also possible that our WG patients had higher rates of hypothermia compared to nonneurosurgical series due to the cranial surgical location and extent of heat loss through this area of the body.

\section{Study Limitations}

Perhaps the most significant limitation of this study is its design: in order to facilitate the evaluation of the warming protocol, we opted to not conduct a randomized study. Although this certainly would have made for a statistical analysis less vulnerable to bias, we believe that the magnitude of the difference is large enough to foster confidence in the result. Additional bias is introduced by use of the tympanic thermometer: as noted above, there is a relatively large confidence interval about core temperatures. We opted for this less than perfect method of temperature measurement in order to facilitate the gathering of data in awake pediatric patients.

\section{Implications for Surgical Quality}

The CMS established the SCIP to reduce SSIs nationally by $10 \%$. The "Inf 10 " section of the SCIP measures calls for maintenance of perioperative normothermia. Hospital compliance with these measures is reported in the SCIP database and is a determinant in hospital reimbursement. It is unclear whether these incentive-based practices change healthcare outcomes, but these programs do increase reportability and compliance with required measures. ${ }^{27}$ SCIP was created in conjunction with the Physicians Quality Reporting Initiative (PQRI), which provides a $2 \%$ Medicare bonus to anesthesia providers for reporting maintenance of normothermia and use of FAWs. ${ }^{24}$ SCIP follows similar projects including the NSQIP and the WHO Surgical Safety Checklist, which are both validated and meaningful measures that have reduced SSIs. ${ }^{27}$ The National Quality Forum has also put forth perioperative temperature management standards. Most metrics relate to reporting or outcomes and few focus on process. ${ }^{2}$ As evidence-based guidelines grow, more metrics relating to process may develop.

Pay-for-performance measures are increasingly more 
important for hospital and individual providers and are an integral part in attempting to provide more value in healthcare. The CMS has delegated $\$ 1.35$ billion to continue a $1.5 \%$ pay-for-reporting bonus, and Medicare has given $\$ 9$ million in incentive-based payments. ${ }^{9}$ At this time normothermia requirements are focused on hospitals and anesthesia providers, but as available evidence for normothermia grows, similar pay-for-performance measures may be applicable to surgeons. The cost for an SSI ranges from $\$ 10$ to $\$ 25,000$ and shunt infections are known to be the most costly SSI of all., ${ }^{, 26} \mathrm{PH}$ prevention strategies are relatively low cost (approximately \$10), safe, and easily implemented. ${ }^{9}$

\section{Conclusions}

Without a specific perioperative warming regimen, $>$ $50 \%$ of pediatric neurosurgery patients experience $\mathrm{PH}$. Preoperative warming appears to reduce the risk of $\mathrm{PH}$ at time 0 intraoperatively as well as insulating patients from developing $\mathrm{PH}$ at the temperature nadir at approximately 45 minutes intraoperatively. It is clear from these data that intraoperative warming protocols reduce both the risk of any hypothermia and the total hypothermic deficit. Only limited resources were required for implementation at a low cost; staff comfort was not significantly affected-although these data were not explicitly collected, only rarely was OR staff behavior affected and complaints were minimal. Resource costs in the US healthcare environment clearly outweigh the risks of increased blood loss, SSIs, and hospital length of stay. Pediatric neurosurgery centers may implement this or similar protocols in order to reduce the hypothermic burden of pediatric neurosurgical patients. Further refinement and improved adherence to this protocol will further reduce the incidence of hypothermia sustained in even WG patients.

\section{References}

1. Beedle SE, Phillips A, Wiggins S, Struwe L: Preventing unplanned perioperative hypothermia in children. AORN J 105:170-183, 2017

2. Benn J, Arnold G, Wei I, Riley C, Aleva F: Using quality indicators in anaesthesia: feeding back data to improve care. Br J Anaesth 109:80-91, 2012

3. Bernard H: Patient warming in surgery and the enhanced recovery. Br J Nurs 22:319-320, 322-325, 2013

4. Berríos-Torres SI, Umscheid CA, Bratzler DW, Leas B, Stone EC, Kelz RR, et al: Centers for Disease Control and Prevention Guideline for the Prevention of Surgical Site Infection, 2017. JAMA Surg 152:784-791, 2017

5. Bräuer A: Perioperative Temperature Management. New York: Cambridge University Press, 2017

6. Gasim GI, Musa IR, Abdien MT, Adam I: Accuracy of tympanic temperature measurement using an infrared tympanic membrane thermometer. BMC Res Notes 6:194, 2013

7. Giuliano KK, Hendricks J: Inadvertent perioperative hypothermia: current nursing knowledge. AORN J 105:453-463, 2017

8. Gustafsson IL, Elmqvist C, From-Attebring M, Johansson I, Rask M: The nurse anesthetists' adherence to Swedish national recommendations to maintain normothermia in patients during surgery. J Perianesth Nurs 32:409-418, 2017

9. Hannenberg AA, Sessler DI: Improving perioperative temperature management. Anesth Analg 107:1454-1457, 2008
10. Hart SR, Bordes B, Hart J, Corsino D, Harmon D: Unintended perioperative hypothermia. Ochsner J 11:259-270, 2011

11. Hoda MR, Popken G: Maintaining perioperative normothermia during laparoscopic and open urologic surgery. J Endourol 22:931-938, 2008

12. Horosz B, Malec-Milewska M: Niezamierzona śródoperacyjna hipotermia. Anestezjol Intens Ter 45:41-47, 2013

13. Kellam MD, Dieckmann LS, Austin PN: Forced-air warming devices and the risk of surgical site infections. AORN J 98:354-369, 2013

14. Kim P, Taghon T, Fetzer M, Tobias JD: Perioperative hypothermia in the pediatric population: a quality improvement project. Am J Med Qual 28:400-406, 2013

15. Kurz A, Sessler DI, Lenhardt R: Perioperative normothermia to reduce the incidence of surgical-wound infection and shorten hospitalization. N Engl J Med 334:1209-1215, 1996

16. Levin RF, Wright F, Pecoraro K, Kopec W: Maintaining perioperative normothermia: sustaining an evidence-based practice improvement project. AORN J 103:213.e1-213.e13, 2016

17. McGovern PD, Albrecht M, Belani KG, Nachtsheim C, Partington PF, Carluke I, et al: Forced-air warming and ultra-clean ventilation do not mix: an investigation of theatre ventilation, patient warming and joint replacement infection in orthopaedics. J Bone Joint Surg Br 93:1537-1544, 2011

18. O'Donnell A: Anaesthesia: A Very Short Introduction. Oxford: Oxford University Press, 2012

19. Pearce B, Christensen R, Voepel-Lewis T: Perioperative hypothermia in the pediatric population: prevalence, risk factors and outcomes. J Anesth Clin Res 1:102, 2010

20. Rajagopalan S, Mascha E, Na J, Sessler DI: The effects of mild perioperative hypothermia on blood loss and transfusion requirement. Anesthesiology 108:71-77, 2008

21. Ryan-Wenger N, Sims M, Patton R, Williamson J: Selection of the most accurate thermometer devices for clinical practice: part 1: meta-analysis of the accuracy of non-core thermometer devices compared to core body temperature. Pediatr Nurs 44:116-133, 2018

22. Sessler DI: Mild perioperative hypothermia. N Engl J Med 336:1730-1737, 1997

23. Sessler DI: Perioperative thermoregulation and heat balance. Lancet 387:2655-2664, 2016

24. Sessler DI: Temperature monitoring: the consequences and prevention of mild perioperative hypothermia. S Afr J Anaesth Analg 20:25-31, 2014

25. Sessler DI: The thermoregulation story. Anesthesiology 118:181-186, 2013

26. Thompson DNP, Hartley JC: Shunt infections, in Albright AL, Pollack IF, Adelson PD (eds): Principles and Practice of Pediatric Neurosurgery, ed 3. New York: Thieme, 2015

27. Tillman M, Wehbe-Janek H, Hodges B, Smythe WR, Papaconstantinou HT: Surgical care improvement project and surgical site infections: can integration in the surgical safety checklist improve quality performance and clinical outcomes? J Surg Res 184:150-156, 2013

28. Torossian A, Bräuer A, Höcker J, Bein B, Wulf H, Horn EP: S3-Leitlinie: Vermeidung von unbeabsichtigter perioperativer Hypothermie. Anasthesiol Intensivmed (Erlangen) 56:308-315, 2015

29. Wong PF, Kumar S, Bohra A, Whetter D, Leaper DJ: Randomized clinical trial of perioperative systemic warming in major elective abdominal surgery. Br J Surg 94:421-426, 2007

30. Young VL, Watson ME: Prevention of perioperative hypothermia in plastic surgery. Aesthet Surg J 26:551-571, 2006

\section{Disclosures}

The authors report no conflict of interest concerning the materi- 
als or methods used in this study or the findings specified in this paper.

\section{Author Contributions}

Conception and design: Mutchnick, Braun, Bohn, Gump,

Moriarty. Acquisition of data: Mutchnick, Braun, Bohn. Analysis and interpretation of data: Mutchnick, Thatikunta, Polivka,

Vickers-Smith. Drafting the article: Mutchnick, Thatikunta. Critically revising the article: Mutchnick, Thatikunta. Reviewed submitted version of manuscript: all authors. Approved the final version of the manuscript on behalf of all authors: Mutchnick. Statistical analysis: Daniels. Administrative/technical/material support: Mutchnick. Study supervision: Mutchnick.

\section{Supplemental Information}

Previous Presentations

Portions of this work have been presented as proceedings at the International Society of Pediatric Neurosurgery, Tel Aviv, Israel, in October 2018, and as a poster at Research!Louisville in Louisville, Kentucky, in October 2015.

\section{Correspondence}

Ian Mutchnick: Norton Children's Hospital, Norton Neuroscience Institute, Louisville, KY. ian.mutchnick@nortonhealthcare.org. 